\title{
COMPOSIÇÃO FLORÍSTICA E GRUPOS ECOLÓGICOS DAS ESPÉCIES DE UM TRECHO DE FLORESTA SEMIDECÍDUA SUBMONTANA DA FAZENDA SÃO GERALDO, VIÇOSA-MG ${ }^{1}$
}

Alexandre Francisco da Silva ${ }^{2}$, Riusson Vitor de Oliveira ${ }^{3}$, Nelson Roberto Loureiro Santos ${ }^{4}$ e Alessandro de Paula ${ }^{5}$

\begin{abstract}
RESUMO - O presente trabalho foi realizado em um trecho de Floresta Estacional Semidecidual Submontana no município de Viçosa (2045’23”S e 4252’23”W), Zona da Mata de Minas Gerais, cujo clima é do tipo Cwa, com os objetivos de determinar a composição florística da vegetação arbórea e inferir acerca dos respectivos grupos ecológicos. A listagem florística foi extraída de um levantamento fitossociológico no qual foram empregados 158 pontos-quadrantes. Foram amostrados 632 indivíduos com circunferência de tronco igual ou maior que $15 \mathrm{~cm}$ à altura de $130 \mathrm{~cm}$ do solo. Foram analisados dez levantamentos realizados na Zona da Mata, para obter as principais espécies ocorrentes na região. Foram determinadas 123 espécies, pertencentes a 85 gêneros e 36 famílias. Devido às proporções de espécies pelos grupos ecológicos, o fragmento florestal estudado foi classificado em estádio médio de regeneração. Dentre as espécies encontradas, sete pertencem à lista de espécies ameaçadas de extinção no Estado de Minas Gerais e no Brasil.
\end{abstract}

Palavras-chave: Florística, sucessão ecológica, Floresta Estacional Semidecidual e grupos ecológicos.

\section{FLORISTIC COMPOSITION AND ECOLOGICAL GROUPS OF SPECIES OF A SUBMONTANE SEMIDECIDUOUS FOREST STRETCH ON SÃO GERALDO FARM, VIÇOSA, MINAS GERAIS - BRAZIL}

\begin{abstract}
This work was carried out in a Submontane Semideciduous Forest fragment stretch on São Geraldo

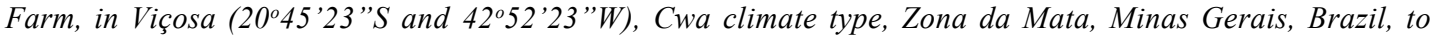
determine the floristic composition of the tree stratum and make some inferences about its respective ecological groups. The floristic listing was taken from a phytosociological survey in which 158 point-centered-quarters were employed. Ten surveys carried on the Zona da Mata were analysed to determine the main species of the region. A total of 632 individuals with a minimum trunk circunference of $0.15 \mathrm{~m}$ at $1.30 \mathrm{~m}$ above the soil were sampled, comprising 123 species, 85 genera and 36 families. According to the ratios of the species distributed over the ecological groups, the fragment under study was classified as being at an intermediary stage of regeneration. Amongst the species observed, some are considered endangered species not only in the state of Minas Gerais but also in Brazil.
\end{abstract}

Key words: $\quad$ Floristic, ecological sucession, Submontane Semideciduous Forest and ecological groups.

\section{INTRODUÇÃO}

Historicamente no Brasil, a vegetação natural vem sendo suprimida para dar lugar, principalmente, à expansão das fronteiras agrícola e pecuária. Na maioria das atividades, novas áreas são abertas, quer seja por esgotamento dos solos ou pela necessidade de maior produção.

1 Recebido para publicação em 9.4.2001.

Aceito para publicação em 12.5.2003.

Trabalho desenvolvido com apoio financeiro da FAPEMIG

2 Dep. de Biologia Vegetal da Universidade Federal de Viçosa - UFV, 36571-000 Viçosa-MG, <afsilva@ufv.br>, ${ }^{3}$ Eng. Florestal - Instituto Estadual de Florestas - MG, ${ }^{4}$ Eng. Florestal, ${ }^{5}$ Eng. Florestal - M.S., Botânica. 
O continuum florestal do Sudeste a que se referiu Aubréville (1959), que na altura do litoral espíritosantense extendia-se para oeste até as cercanias de Belo Horizonte, foi retalhado pelo desflorestamento intensivo, a partir da metade do século XIX, para implantação de monoculturas, especialmente do café, e formação de pastos para criação de gado em regime extensivo (Paniago, 1983). Essas atividades alteraram profundamente a paisagem da Zona da Mata de Minas Gerais.

A região de Viçosa apresenta uma paisagem que mostra o confinamento das florestas em fragmentos, geralmente em locais de difícil acesso à explotação ou guardados por propriedades particulares, os quais, embora pequenos, são importantes porque ainda mantêm representantes da flora original, além de serem fontes de diásporas, que podem servir à recuperação e ao enriquecimento de áreas depauperadas, haja vista o alto grau de conectividade entre eles.

Entre as várias conseqüências resultantes do processo de fragmentação das florestas tropicais citadas por Viana et al. (1992), destacam-se o distúrbio do regime hidrológico das bacias hidrográficas e as mudanças climáticas. Entretanto, o aspecto resultante de maior gravidade é a perda da biodiversidade.

Ainda são poucos os trabalhos de levantamento dos recursos vegetais na Zona da Mata mineira (Soares Júnior, 2000). Portanto, este trabalho teve como objetivos determinar a composição florística do estrato arbóreo, classificar as espécies nos respectivos grupos ecológicos e levantar, dentro destes grupos, as espécies mais freqüentemente encontradas na região que possam ser indicadas para atividades de recomposição e enriquecimento de áreas.

\section{MATERIAIS E MÉTODOS}

O presente trabalho foi desenvolvido em uma área de floresta secundária da Formação de Floresta Estacional Semidecidual Submontana (Oliveira Filho et al., 1994), protegida permanentemente, no município de Viçosa, Minas Gerais $\left(20^{\circ} 45^{\prime} 23^{\prime \prime} \mathrm{S}\right.$ e $\left.42^{\circ} 52^{\prime} 23^{\prime \prime} \mathrm{W}\right)$, em altitude de $670 \mathrm{~m}$, com relevo variando de ondulado a montanhoso.

O clima da região é do tipo Cwa, mesotérmico úmido com verões chuvosos e invernos secos, segundo a classificação de Köppen, apresentando déficit hídrico no período de maio a setembro e um excedente entre

R. Árvore, Viçosa-MG, v.27, n.3, p.311-319, 2003 dezembro e março, sendo a precipitação pluvial média anual de $1.248 \mathrm{~mm}$ e a umidade anual relativa média do ar de 80,6\% (Soares Júnior, 2000). A temperatura média anual do ar é de $19{ }^{\circ} \mathrm{C}$, sendo a máxima de $26,1^{\circ} \mathrm{C}$ e a mínima de $14{ }^{\circ} \mathrm{C}$ (Castro et al., 1973).

O fragmento objeto deste trabalho, com 32 ha, localiza-se na fazenda São Geraldo, cujo histórico é conhecido a partir de 1973. Desde então, a mata vem sendo preservada e tratada como Reserva Biológica, com proibição de caça e corte de árvores, contando, para tanto, com o apoio da Polícia Florestal.

A listagem florística foi extraída de um trabalho fitossociológico, para o qual foram empregados 158 pontos-quadrantes (Cottam \& Curtis, 1956), com as modificações propostas por Martins (1993), para indivíduos arbóreos que possuíam perímetro de tronco mínimo de $15 \mathrm{~cm}$ a altura a $130 \mathrm{~cm}$ do solo (CAP).

Os trabalhos de identificação taxonômica foram realizados no Laboratório de Taxonomia do Herbário do Departamento de Biologia Vegetal da Universidade Federal de Viçosa (VIC). A grafia das espécies e as respectivas autoridades estão de acordo com o INDEX KEWENSIS (1997) e, em alguns casos, com bibliografia mais recente.

Fêz-se um levantamento das espécies arbóreas mais freqüentemente encontradas nos fragmentos florestais da Zona da Mata, indicando os respectivos grupos ecológicos, com vistas a trabalhos de recuperação ambiental. Além da listagem do presente trabalho, foram consultados os trabalhos de: Abreu (1997), Almeida \& Souza (1997), Marangon (1999), Meira Neto et al. (1997), Meira Neto \& Martins (2000), Sevilha et al. (2001), Silva et al. (2000), Soares Júnior (2000) e Senra (2000).

\section{RESULTADOS E DISCUSSÃO}

Dos 632 indivíduos amostrados, foram encontradas 123 espécies, pertencentes a 85 gêneros e 36 famílias (Quadro 1). Esses dados mostram riqueza compatível a outros realizados em Florestas Estacionais Semideciduais da Zona da Mata.

Os gêneros mais bem representados em número de espécie foram Casearia com cinco espécies, Machaerium e Trichilia com quatro espécies cada um e Croton, Guatteria, Myrcia, Ocotea, Rollinia e Solanum com três cada um. 


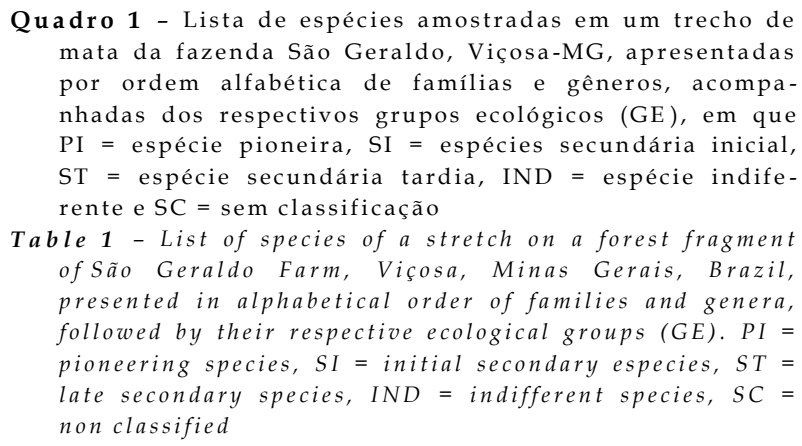
mata da fazenda São Geraldo, Viçosa-MG, apresentadas por ordem alfabética de famílias e gêneros, acompanhadas dos respectivos grupos ecológicos (GE), em que $\mathrm{PI}=$ espécie pioneira, $\mathrm{SI}=$ espécies secundária inicial, $\mathrm{ST}=$ espécie secundária tardia, $\mathrm{IND}=$ espécie indiferente e $\mathrm{SC}=$ sem classificação

Table 1 - List of species of a stretch on a forest fragment of São Geraldo Farm, Viçosa, Minas Gerais, Brazil, presented in alphabetical order of families and genera, followed by their respective ecological groups (GE). $P I=$ pioneering species, $S I=$ initial secondary especies, $S T=$ late secondary species, $I N D=$ indifferent species, $S C=$ non classified

\begin{tabular}{|c|c|}
\hline Táxons & GE \\
\hline $\begin{array}{l}\text { ANACARDIACEAE } \\
\text { Astronium fraxinifolium Schott }\end{array}$ & ST \\
\hline ANNONACEAE & \\
\hline Guatteria australis A.St.-Hil. & SI \\
\hline Guatteria nigrescens Mart. & ST \\
\hline Guatteria villosissima A.St.-Hil. & SI \\
\hline Rollinia laurifolia Schltdt. & SI \\
\hline Rollinia silvatica (A.St.-Hil.)Mart. & SI \\
\hline Rollinia sericea (R.E.Fries) R.E.Fries & ST \\
\hline Xylopia sericea A.St.-Hil. & SI \\
\hline APOCYNACEAE & \\
\hline Tabernaemontana laeta Mart. & PI \\
\hline AQUIFOLIACEAE & \\
\hline Ilex cerasifolia Reissek & ST \\
\hline ARALIACEAE & \\
\hline $\begin{array}{l}\text { Shefflera morototoni (Aubl.) Maquire, Steyerm. } \\
\text { \&Frodin }\end{array}$ & SI \\
\hline ASTERACEAE & \\
\hline Vernonia diffusa Less. & PI \\
\hline BIGNONIACEAE & \\
\hline Jacaranda macrantha Cham. & SI \\
\hline Sparattosperma leucanthum (Vell.) K.Schum. & SI \\
\hline BOMBACACEAE & \\
\hline Eriotheca candolleana (K. Shum.) A. Robyns & SI \\
\hline Pseudobombax grandiflorum (Cav.) A. Robyns & SI \\
\hline BURSERACEAE & \\
\hline Protium warmingianum March. & ST \\
\hline Protium widgrenii Engl. & ST \\
\hline CANNELACEAE & \\
\hline Cinnamodendron sp. & $\mathrm{SC}$ \\
\hline CECROPIACEAE & \\
\hline Cecropia hololeuca Miq. & PI \\
\hline CELASTRACEAE & \\
\hline Maytenus floribunda Reissek & ST \\
\hline Maytenus aquifolium Mart. & $\mathrm{SC}$ \\
\hline CHRYSOBALANACEAE & \\
\hline Licania spicata Hook. f. & SI \\
\hline
\end{tabular}

Quadro 1, cont.

Table 1, cont.

\begin{tabular}{|c|c|}
\hline Táxons & GE \\
\hline $\begin{array}{l}\text { ERYTHROXYLACEAE } \\
\quad \text { Erythroxylum pelleterianum A. St.-Hil. }\end{array}$ & IND \\
\hline EUPHORBIACEAE & \\
\hline Alchornea glandulosa Poepp. \& Endl.. & SI \\
\hline Alchornea triplinervia Müll.Arg. & SI \\
\hline Croton floribundus Spreng. & PI \\
\hline Croton gracilipes Baill. & PI \\
\hline Croton urucurana Baill. & PI \\
\hline Hieronyma alchorneoides Allemão & SI \\
\hline Mabea fistulifera Mart. & PI \\
\hline Maprounea guianensis Aubl. & SI \\
\hline Pera glabrata Poepp. ex Baill. & SI \\
\hline Sapium glandulosum (L.) Morong & SI \\
\hline FLACOURTIACEAE & \\
\hline Carpotroche brasiliensis (Raddi) Endl. & ST \\
\hline Casearia arborea Urb. & SI \\
\hline Casearia decandra Jacq. & SI \\
\hline Casearia sylvestris Sw. & SI \\
\hline Casearia ulmifolia Cambess. & SI \\
\hline Casearia gossypiosperma Briq. & $\mathrm{SC}$ \\
\hline GUTTIFERAE & \\
\hline Rheedia gardneriana Planch. \& Triana & ST \\
\hline Vismia martiana H. G. Reich. & PI \\
\hline LACISTEMACEAE & \\
\hline Lacistema pubescens Mart. & SI \\
\hline LAURACEAE & \\
\hline Endlicheria paniculata (Spreng.) J. F. Macbr. & ST \\
\hline Licaria armeniaca (Nees)Kosterman & ST \\
\hline Nectandra lanceolata Nees & ST \\
\hline Nectandra oppositifolia Nees & SI \\
\hline Ocotea dispersa $\mathrm{Mez}$ & ST \\
\hline Ocotea odorifera (Vell.)Rohwer & ST \\
\hline Ocotea tenuiflora $\mathrm{Mez}$ & ST \\
\hline LECYTHIDACEAE & \\
\hline Cariniana estrellensis Kuntze & ST \\
\hline Cariniana legalis Kuntze & ST \\
\hline LEGUMINOSAE Caesalpinioideae & \\
\hline Apuleia leiocarpa J. F. Macbr. & SI \\
\hline Bauhinia forficata Link & SI \\
\hline Cassia ferruginea Schrad. ex. DC. & SI \\
\hline Copaifera langsdorffii Desf. & SI \\
\hline Melanoxylon brauna Schott & ST \\
\hline Peltophorum dubium Taub. & SI \\
\hline Pterogyne nitens Tul. & SI \\
\hline Senna silvestris (Vell.) Irwin \& Barneby & SI \\
\hline
\end{tabular}


Quadro 1, cont.

Table 1, cont.

\begin{tabular}{|c|c|}
\hline Táxons & GE \\
\hline \multicolumn{2}{|l|}{ LEGUMINOSAE Mimosoideae } \\
\hline $\begin{array}{l}\text { Anadenanthera colubrina (Vell.) Brenan var. cebil } \\
\text { (Griseb.) Altschul }\end{array}$ & SI \\
\hline Inga alba (Sw.)Willd. & SI \\
\hline Inga sessilis Mart. & PI \\
\hline $\begin{array}{l}\text { Pseudopiptadenia contorta (DC.) G. P. Lewis \& } \\
\text { M.P.Lima }\end{array}$ & SI \\
\hline Piptadenia gonoacantha J.F. Macbr. & PI \\
\hline Piptadenia paniculata Benth. & PI \\
\hline Plathymenia foliolosa Benth. & SI \\
\hline \multicolumn{2}{|l|}{ LEGUMINOSAE Papilionoideae } \\
\hline Andira fraxinifolia Benth. & SI \\
\hline Dalbergia nigra Allemão ex Benth. & SI \\
\hline Lonchocarpus guilleminianus (Tul.) Malme & ST \\
\hline Machaerium brasiliense Vogel & SI \\
\hline Machaerium floridum (Mart.) Ducke & SI \\
\hline Machaerium nyctitans (Vell.) Benth. & PI \\
\hline Machaerium stipitatum (DC.) Vogel & SI \\
\hline \multicolumn{2}{|l|}{ MALPIGHIACEAE } \\
\hline Bunchosia pallescens Skottsb. & SI \\
\hline \multicolumn{2}{|l|}{ MELASTOMATACEAE } \\
\hline Miconia calvescens DC. & $\mathrm{SC}$ \\
\hline \multicolumn{2}{|l|}{ MELIACEAE } \\
\hline Guarea guidonia (L.) Sleumer & ST \\
\hline Guarea macrophylla Vahl & ST \\
\hline Trichilia catigua A. Juss. & SI \\
\hline Trichilia lepidota $\mathrm{Sw}$. & ST \\
\hline Trichilia pallida Sw. & SI \\
\hline Trichilia riedelli C. DC. & ST \\
\hline \multicolumn{2}{|l|}{ MONIMIACEAE } \\
\hline Siparuna guianensis Aubl. & IND. \\
\hline Siparuna reginae (Tul.) A. DC. & IND. \\
\hline \multicolumn{2}{|l|}{ MORACEAE } \\
\hline Brosimum glaziovii Taub. & SI \\
\hline Ficus enormis (Mart. ex Miq.) Miq. & ST \\
\hline Ficus mexiae Standley & ST \\
\hline Sorocea bonplandii (Baill.) Burger, Lanj. \& Boer & SI \\
\hline $\begin{array}{l}\text { MYRISTICACEAE } \\
\quad \text { Virola oleifera }(\text { Schott) A. C. Sm. }\end{array}$ & SI \\
\hline
\end{tabular}

Quanto ao número de gêneros, as famílias mais bem representadas foram Leguminosae com 16, Rubiaceae com oito, Euphorbiaceae e Myrtaceae com sete cada uma e Lauraceae com quatro.

As famílias mais ricas foram Leguminosae com 22 espécies, Euphorbiaceae com dez, Myrtaceae e
Quadro 1, cont.

Table 1, cont.

\begin{tabular}{|c|c|}
\hline Táxons & $\mathrm{GE}$ \\
\hline \multicolumn{2}{|l|}{ MYRTACEAE } \\
\hline Campomanesia xanthocarpa Berg & ST \\
\hline Eugenia stictosepala Kiaersk. & ST \\
\hline Marlierea sp. & $\mathrm{SC}$ \\
\hline Myrcia fallax DC. & SI \\
\hline Myrcia sphaerocarpa DC. & ST \\
\hline Myrcia sp. & $\mathrm{SC}$ \\
\hline Myrciaria floribunda Berg & SI \\
\hline Plinia glomerata (Berg) Amshoff & ST \\
\hline Psidium guianense Pers. & ST \\
\hline \multicolumn{2}{|l|}{ NYCTAGINACEAE } \\
\hline Guapira opposita (Vell.) Reitz & SI \\
\hline Guapira tomentosa (Casar.) Lundell & SI \\
\hline \multicolumn{2}{|l|}{ RUBIACEAE } \\
\hline Amaioua guianensis Aubl. & SI \\
\hline Bathysa cuspidata Hook. f. & SI \\
\hline Bathysa nicholsonii $\mathrm{K}$. Schum. & SI \\
\hline Coutarea hexandra K. Schum. & SI \\
\hline Guettarda viburnoides Cham.\& Schltdl. & SI \\
\hline Ixora gardneriana Benth. & ST \\
\hline Palicourea guianensis Aubl. & ST \\
\hline Psychotria sessilis Müll. Arg. & SI \\
\hline Rudgea lanceolata Benth. & SI \\
\hline \multicolumn{2}{|l|}{ RUTACEAE } \\
\hline Hortia arborea Engl. & ST \\
\hline Zanthoxylum rhoifolium Lam. & PI \\
\hline \multicolumn{2}{|l|}{ SAPINDACEAE } \\
\hline Allophylus edulis Radlk. ex Warm. & SI \\
\hline Allophylus sericeus Radlk. & SI \\
\hline Cupania tenuivalvis Radlk. & SI \\
\hline Cupania vernalis Cambess. & SI \\
\hline Matayba elaeagnoides Radlk. & SI \\
\hline Matayba guianensis Aubl. & SI \\
\hline \multicolumn{2}{|l|}{ SAPOTACEAE } \\
\hline Chrysophyllum gonocarpum Engl. & ST \\
\hline Chrysophyllum sp. & $\mathrm{SC}$ \\
\hline \multicolumn{2}{|l|}{ SOLANACEAE } \\
\hline Cestrum amictum Schltdl. & PI \\
\hline Solanum bullatum Vell. & PI \\
\hline Solanum cernuиm Vell. & PI \\
\hline Solanum leucodendron Sendt. & $\mathrm{SC}$ \\
\hline
\end{tabular}

Rubiaceae com nove, Annonaceae e Lauraceae com sete e Flacourtiaceae, Meliaceae e Sapindaceae, com seis cada uma.

Sete espécies encontradas na amostragem pertencem à lista de espécies ameaçadas de extinção no Estado de Minas Gerais (FUNDAÇÃO..., 2000). Três delas estão 
relacionadas na categoria "vulnerável" Guatteria villosissima (Annonaceae), Melanoxylon brauna (Leguminosae Caesalpinioideae, Dalbergia nigra (Leguminosae Faboideae), devendo ser ressaltado que as duas últimas estão presentes também na Lista Oficial do Ibama (IBAMA, 1992). Na categoria "em perigo" está Ocotea odorifera (Lauraceae), que também faz parte da Lista Oficial do Ibama. Já na lista das espécies "presumivelmente ameaçadas de extinção" estão relacionadas Guatteria australis (Annonaceae), Vernonia diffusa (Asteraceae) e Guapira tomentosa (Nyctaginaceae).

No Quadro 1 está a classificação proposta para as espécies encontradas no presente trabalho, no qual foram reunidas informações dos trabalhos de Gandolfi et al. (1995), Almeida (1996), Ferretti et al. (1995) e Paula (1999). No entanto, a classificação apresentada neste trabalho pode, em alguns casos, diferir daqueles autores, quando as observações de campo realizadas na região assim tenham indicado.

A classificação seguiu a proposta de Gandolfi et al. (1995), que classificaram as espécies em pioneiras, secundárias iniciais e secundárias tardias, sendo os grupos relacionados às três categorias de sucessão: fase inicial, média e avançada de sucessão, respectivamente.

Entre as espécies secundárias tardias do dossel foram encontradas Astronium fraxinifolium, Cariniana estrellensis, C. legalis, Endlicheria paniculata, Ficus enormis, F. mexiae, Ilex cerasifolia, Lonchocarpus guilleminianus, Nectandra lanceolata, Ocotea odorifera, O. tenuifolia e Trichilia lepidota, enquanto entre as espécies de submata destacam-se Campomanesia xanthocarpa, Carpotroche brasiliensis, Chrysophyllum gonocarpum, Eugenia stictosepala, Guarea macrophylla, G. Guidonia, Guatteria nigrescens, Hortia arborea, Ixora gardneriana, Maytenus aquifolium, Plinia glomerata, Protium warmingianum e Rheedia gardneriana.

Entre as espécies consideradas secundárias iniciais que permanecem no dossel da floresta, que correspondem às clímax heliófilas de Swaine \& Whitmore (1988), foram encontradas Apuleia leiocarpa, Alchornea triplinervia, Allophylus edulis, A. sericeus, Anadenanthera colubrina, Cassia ferruginea, Copaifera langsdorffii, Hieronyma alchorneoides, Melanoxylon brauna, Schefflera morototoni, Pseudopiptadenia contorta, Sapium glandulosum, Machaerium floridum, M. stipitatum e Plathymenia foliolosa, enquanto na submata foram encontradas Eriotheca candolleana, Dalbergia nigra,
Guatteria australis, G. villosissima, Myrcia fallax, Rollinia silvatica, Protium widgrenii, Sparattosperma leucanthum, Trichilia catigua, T. pallida, Xylopia sericea, entre outras.

Algumas espécies, embora pioneiras, alcançam o dossel e permanecem à luz mesmo depois do maior desenvolvimento da floresta, das quais encontram-se indivíduos jovens nas áreas com grande penetração de luz. Entre estas destacaram-se Cecropia hololeuca, Machaerium nyctitans, Piptadenia gonoacantha, P. paniculata e Zanthoxylum rhoifolium, enquanto entre as típicas das clareiras maiores e das bordas encontraram-se Cestrum amictum, Croton floribundus, C. gracilipes, C. urucurana, Mabea fistulifera, Solanum bullatum, Solanum cernuum, Vernonia diffusa e Vismia martiana.

Analisando o número de espécies de cada grupo ecológico, a mata da fazenda São Geraldo pode ser classificada em estádio médio de sucessão secundária, em franco desenvolvimento para a fase madura. Esta suposição parte da constatação de que existe cerca de $63 \%$ de espécies secundárias iniciais, $27 \%$ de secundárias tardias e $10 \%$ de pioneiras, o que indica uma tendência à maior adequação das condições para o sucesso no recrutamento de espécies tardias.

Para alguns autores os fragmentos florestais apresentam-se na forma de mosaico vegetacional, advindo de distúrbios naturais ou provocados. Nesse mosaico podem ser reconhecidas as fases de clareira, a de construção e a madura, que estão sujeitas a mudanças temporais próprias dos processos dinâmicos das comunidades (Spurr, 1952; Swaine \& Whitmore, 1988). Por outro lado, Rollet (1983a, b) contestou a teoria do mosaico, afirmando ser esta uma situação excepcional.

A classificação de espécies nos respectivos grupos ecológicos tem esbarrado em dois fatores primordiais. $\mathrm{O}$ primeiro é que os critérios utilizados diferem entre autores, o que leva algumas espécies a serem classificadas em grupos distintos. O segundo refere-se ao fato de que uma mesma espécie, dependendo de suas características genéticas, pode responder de forma diferente, diante das condições ambientais ocorrentes em regiões com solos e climas distintos, uma vez que estas respostas não se dão para um único fator do meio isoladamente.

O nível de luminosidade que chega ao chão da floresta é um dado importante. Neste aspecto, o grau de caducifolia da floresta ou em trechos dela interfere no recrutamento de espécies (Gandolfi et al., 1995). Um 
Quadro 2 - Espécies arbóreas mais freqüentemente encontradas em dez levantamentos realizados na Zona da Mata de Minas Gerais. Na coluna um (GE) consta o grupo ecológico, em que PI = pioneira, SI = secundária inicial, ST = secundária tardia e IND = indiferente. Nas demais colunas, estão assinalados os números de levantamentos nos quais foi encontrada a espécie

Table 2 - Tree species most frequently found in ten forest fragments of Zona da Mata, Minas Gerais, Brazil, presented in alphabetical order, followed by their respective ecological groups $(G E)$. PI = pioneering species, SI = initial secondary especies, $S T=$ late secondary species, IND = indifferent species, $S C=$ non classified. In subsequent columns, the survey numbers in which each species was found are shown

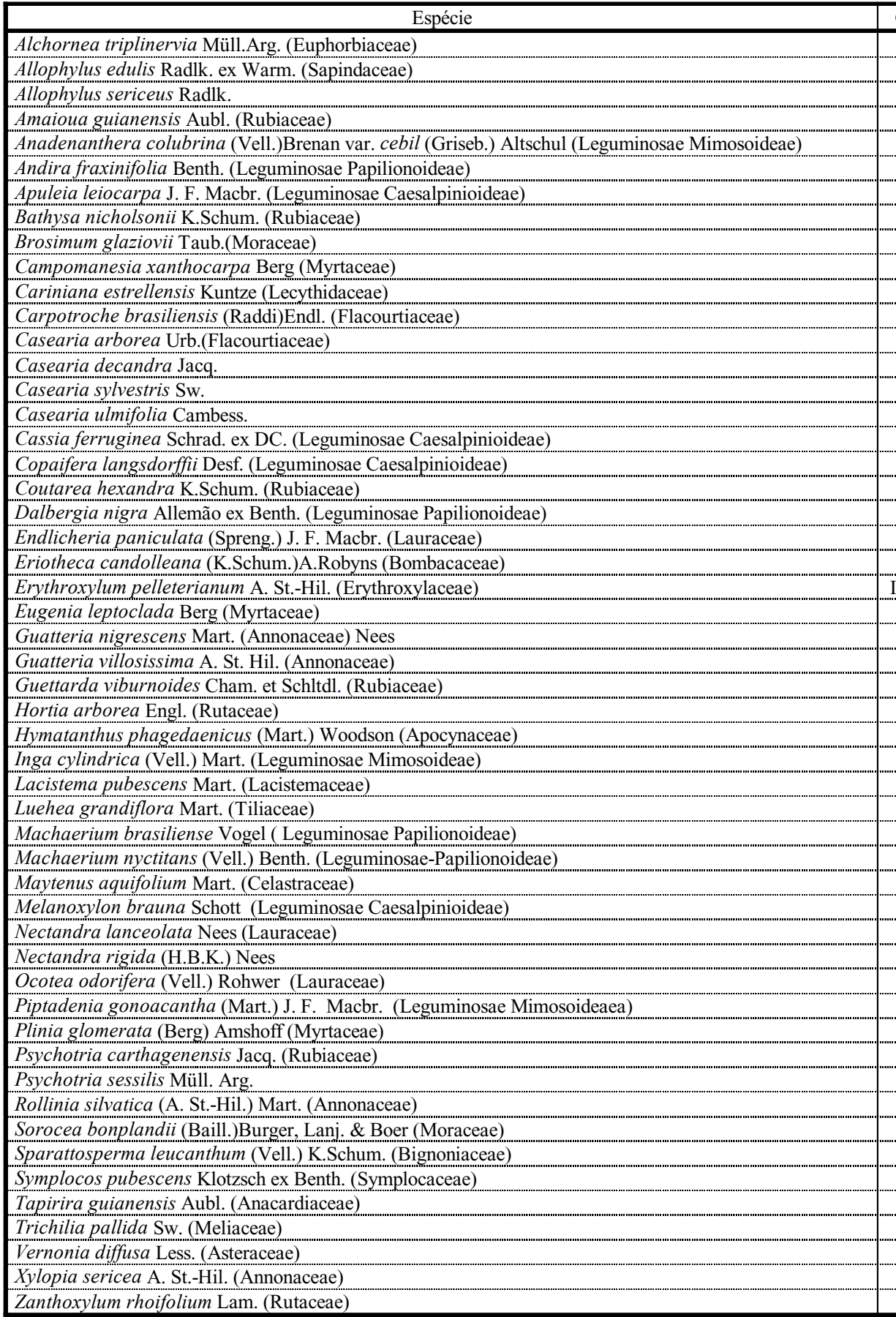

\begin{tabular}{|c|c|c|c|c|c|}
\hline GE & 9 & 8 & 7 & 6 & 5 \\
\hline SI & & & & & $X$ \\
\hline SI & & $\mathrm{X}$ & & & \\
\hline SI & & & & X & \\
\hline SI & & & $X$ & & \\
\hline SI & & & & X & \\
\hline SI & & & & & $\mathrm{X}$ \\
\hline SI & $X$ & & & & \\
\hline SI & X & & & & \\
\hline SI & & & $X$ & & \\
\hline ST & & & & X & \\
\hline ST & & & & & $\mathrm{X}$ \\
\hline ST & & & & X & \\
\hline SI & & $X$ & & & \\
\hline SI & & $\mathrm{X}$ & & & \\
\hline SI & & & & & \\
\hline SI & & $X$ & & & \\
\hline SI & & & & $X$ & \\
\hline SI & & $X$ & & & \\
\hline SI & & & & & $\mathrm{X}$ \\
\hline SI & $X$ & & & & \\
\hline ST & & & $X$ & & \\
\hline SI & & & & & $\mathrm{X}$ \\
\hline IND & & $\mathrm{X}$ & & & \\
\hline ST & & & $X$ & & \\
\hline ST & & & & & $\mathrm{X}$ \\
\hline SI & & & & & $\mathrm{X}$ \\
\hline SI & & & & X & \\
\hline ST & & $X$ & & & \\
\hline ST & & & & X & \\
\hline SI & & & & & $\mathrm{X}$ \\
\hline SI & & & $X$ & & \\
\hline PI & & & & & $X$ \\
\hline SI & & & & & \\
\hline PI & & $X$ & & & \\
\hline ST & & & & & $\mathrm{X}$ \\
\hline ST & & & & X & \\
\hline ST & & & & & $\mathrm{X}$ \\
\hline SI & & & & & $x$ \\
\hline ST & $X$ & & & & \\
\hline PI & $X$ & & & & \\
\hline ST & & & & & $\mathrm{X}$ \\
\hline ST & & & & $\mathrm{X}$ & \\
\hline SI & & & & $\mathrm{X}$ & \\
\hline SI & & & X & & \\
\hline SI & $\mathrm{X}$ & & & & \\
\hline SI & & & & X & \\
\hline SI & & & & & $X$ \\
\hline SI & & & & $\mathrm{X}$ & \\
\hline SI & & & & X & \\
\hline PI & & $\mathrm{X}$ & & & \\
\hline SI & & & & $X$ & \\
\hline PI & & $\mathrm{X}$ & & & \\
\hline
\end{tabular}


outro dado importante foi destacado por Canham (1989) para florestas temperadas, nas quais haveria, entre espécies tolerantes à sombra, um gradiente de respostas qualitativas e quantitativas em relação ao aumento da luminosidade, advindo da abertura do dossel nas clareiras, que mesmo quando de pequenas dimensões aumentaria significativamente a luminosidade no sub-bosque, uma vez que a área afetada poderia se estender por áreas muito maiores do que a da clareira. Para florestas tropicais há registros que relatam existir nas clareiras pequenas mais espécies secundárias tardias do que secundárias iniciais e pioneiras (Whitmore, 1989; Costa \& Mantovani, 1992; Tabarelli, 1997; Tabarelli \& Mantovani; 1997; Martins, 1999).

Também de importância para o recrutamento de espécies é a face de exposição do fragmento, bem como sua situação no relevo, que pode, devido à intensidade e qualidade da luz recebida ao longo do ano, interferir na quantidade de água disponível no solo, ou se o fragmento encontra-se sobre sítio plano ou movimentado.

Assim, pode-se depreender que são muitos os fatores que intervêm no sucesso do recrutamento de espécies, daí a importância de se buscar dados que possam auxiliar na compreensão do comportamento ecológico das espécies.

O Quadro 2 apresenta as espécies arbóreas mais comumente encontradas nos fragmentos florestais da Zona da Mata, com seus respectivos grupos ecológicos, que poderiam ser utilizadas em projetos de recuperação de áreas degradadas e enriquecimento de fragmentos em desenvolvimento. Em todos os levantamentos analisados estiveram presentes Mabea fistulifera (pioneira), Guapira opposita e Matayba elaeagnoides, (secundárias iniciais) e Siparuna guianensis (espécie indiferente).

Espécies raramente encontradas merecem atenção especial sob o ponto de vista conservacionista. Entre as espécies arbóreas, foram exclusivamente encontradas no presente trabalho Bathysa cuspidata, Bunchosia pallescens, Croton gracilipes, Ficus mexiae, Maytenus floribunda, Ocotea tenuiflora, Psidium guianense, Senna silvestris, Solanum bullatum e Trichilia riedelli.

Entre as espécies encontradas no máximo em três dos dez levantamentos analisados para a Zona da Mata estiveram Bauhinia forficata, Cariniana legalis, Cestrum amictum, Cupania tenuivalvis, Eugenia stictosepala, Ficus enormis, Guapira tomentosa, Guarea guidonia, Hieronyma alchorneoides, Inga alba, Matayba guianensis, Miconia calvescens, Myrcia sphaerocarpa, Palicourea guianensis, Pera glabrata, Piptadenia paniculata, Plathymenia foliolosa, Protium widgrenii, Rudgea lanceolata, Shefflera morototoni, Siparuna reginae, Solanum leucodendron e Virola oleifera.

\section{CONCLUSÕES}

A análise do número de espécies de cada grupo ecológico indica que a mata da fazenda São Geraldo pode ser classificada em estádio médio de sucessão secundária, em franco desenvolvimento para a fase madura.

O processo de fragmentação pelo qual passou a Zona da Mata de Minas Gerais e a contínua degradação a que estão submetidos os remanescentes de Florestas Estacionais já justificariam a proteção do fragmento em questão. Além disto, a riqueza encontrada, a existência de várias espécies consideradas raras na região e a presença das espécies Guatteria villosissima, Melanoxylon brauna, Dalbergia nigra, Ocotea odorifera Guatteria australis, Vernonia diffusa e Guapira tomentosa, listadas como ameaçadas ou presumivelmente ameaçadas de extinção no País e no Estado de Minas Gerais, demonstram a importância deste fragmento florestal para a manutenção da riqueza florística da região.

\section{REFERÊNCIAS BIBLIOGRÁFICAS}

ABREU, L. C. M. Composição florística e estrutura fitossociológica de um fragmento florestal no Jardim Botânico da Universidade Federal de Viçosa - Face sul, Viçosa (MG). 1997. 27 f. Monografia (Graduação em Engenharia Florestal) - Universidade Federal de Viçosa, Viçosa, 1997.

ALMEIDA, D. S. Florística e estrutura de um fragmento de Floresta Atlântica, no município de Juiz de Fora, Minas Gerais. 1996. 91 f. Dissertação (Mestrado em Ciência Florestal) - Universidade Federal de Viçosa, Viçosa, 1996.

ALMEIDA, D. S.; SOUZA, A. L. Florística e estrutura de um fragmento de Floresta Atlântica, no município de Juiz de Fora, Minas Gerais. Revista Árvore, v. 21, n. 2, p. 221230, 1997

AUBRÉVILLE, A. As florestas do Brasil - estudo fitogeográfico florestal. Anuário Brasileiro de Economia Florestal, 11, p. 210-232, 1959.

CANHAM, C. D. Different responses to gaps among shadetolerant tree species. Ecology, n. 70, n. 3, p. 548-550, 1989.

R. Árvore, Viçosa-MG, v.27, n.3, p.311-319, 2003 
CASTRO, P. S. et al. Interceptação da chuva por mata natural secundária na região de Viçosa, MG. Revista Árvore, v. 7, n. 1, p. 76-88, 1973.

COSTA, M. P.; MANTOVANI, W. Composição florística e estrutura de clareiras em mata mesófila na Bacia de São Paulo. In: CONGRESSO NACIONAL SOBRE ESSÊNCIAS NATIVAS, 2., 1992, São Paulo. Anais... Revista do Instituto Florestal 4, p. 178-183, 1992. (Edição Especial, parte 1).

COTTAM, G.; CURTIS, J. T. The use of distance measures in phytosociological sampling. Ecology, v. 37, p. 451-460, 1956.

FERRETTI, A. R. et al. Classificação das espécies arbóreas em grupos ecofisiológicos para revegetação com nativas no estado de São Paulo. Florestar Estatístico, v. 3, n. 7, p. 7377, 1995 .

\section{FUNDAÇÃO BIODIVERSITAS, FUNDAÇÃO} ZOOBOTÂNICA DE BELO HORIZONTE. Lista vermelha das espécies ameaçadas de extinção da flora do estado de Minas Gerais. Organizadores: Míriam Pimentel Mendonça e Lívia Vanucci Lins. Belo Horizonte: 2000. $160 \mathrm{p}$.

GANDOLFI, S.; LEITÃO FILHO, H. F.; BEZERRA, C. L. E. Levantamento florístico e caráter sucessional das espécies arbustivo arbóreas de uma floresta mesófila semidecídua no município de Guarulhos, SP. Revista Brasileira de Biologia, v. 55, n. 4, p. 753-767, 1995.

IBAMA. Lista Oficial das Espécies da Flora Brasileira Ameaçadas de Extinção. Diário Oficial. Portaria 006/92-N de 15 de janeiro de 1992. 1992.

INDEX KEWENSIS. Plantarum Phanerogamarum. Oxford: Herbarium of the Botanical Gardens Kew, 1997.

MARANGON, L. C. Florística e fitossociologia de área de Floresta Estacional semidecidual visando dinâmica de espécies florestais arbóreas no município de Viçosa, MG. 1999. 135 f. (Doutorado em Ecologia e Recursos Naturais) - Universidade Federal de São Carlos, São Carlos, 1999.

MARTINS, F. R. Estrutura de uma floresta mesófila. Campinas: UNICAMP, 1993. 246 p.

MARTINS, S. V. Aspectos da dinâmica de clareiras em uma Floresta Estacional Semidecidual no município de Campinas, SP. Campinas, SP. 1999. 214f. Tese (Doutorado em Ciências) - Universidade Estadual de Campinas, Campinas, 1999.

R. Árvore, Viçosa-MG, v.27, n.3, p.311-319, 2003
MEIRA NETO, J. A. A. et al. Estrutura de uma Floresta Estacional Semidecidual Submontana em área diretamente afetada pela usina hidrelétrica de Pilar, Ponte Nova, Zona da Mata de Minas Gerais. Revista Árvore, v. 21, n. 3, p. 337344, 1997.

MEIRA NETO, J. A. A.; MARTINS, F. R. Composição florística do estrato herbáceo-arbustivo de uma Floresta Estacional Semidecidual em Viçosa-MG. Revista Árvore, v. 24, n. 4, p. 407-416, 2000.

OLIVEIRA-FILHO, A. T. et al. Comparison of the woody flora and soils of six areas of Montane Semideciduous Forest in southern Minas Gerais, Brasil. Edinburgh Journal of Botany, v. 51, n. 3, p. 355-389, 1994.

PANIAGO, M. C. T. Evolucão histórica e tendências de mudanças sócio-culturais na comunidade de Viçosa-MG. 1983. 407 f. Dissertação (Dissertação em Economia Rural). Universidade Federal de Viçosa, Viçosa, 1983.

SEVILHA, A. C. et al. Fitossociologia do estrato arbóreo de um trecho de Floresta Estacional Semidecidual no Jardim Botânico da Universidade Federal de Viçosa ( Face Sudoeste), Viçosa (MG). Revista Árvore, v. 25, n. 4, p. 431-443, 2001

PAULA, A. Alterações florísticas e fitossociológicas da vegetação arbórea numa Floresta Estacional Semidecidual em Viçosa-MG. 1999. 87 f. Dissertação (Mestrado em Botânica) - Universidade Federal de Viçosa, Viçosa, 1999.

ROLLET, B. La régénération naturelle dans le trouées: Un processus général de la dynamique des forêts tropicales humides (premiére partie). Revue Bois et Forêts des Tropiques, v. 201, p. 3-34, 1983a.

ROLLET, B. La régénération naturelle dans le trouées: Un processus général de la dynamique des forêts tropicales humides (seconde partie). Revue Bois et Forêts des Tropiques, v. 202, p. 19-34, 1983 b.

SENRA, L. C. Composição florística e estrutura fitossociológica de um fragmento florestal da Fazenda Rancho Fundo, Na Zona da Mata - Viçosa, MG. 2000. 78 f. Dissertação (Mestrado em Botânica) - Universidade Federal de Viçosa, Viçosa, 2000.

SILVA, A. F.; FONTES, N. R. L.; LEITÃO FILHO, H. F. Composição florística e estrutura horizontal do estrato arbóreo de um trecho da Mata da Biologia da Universidade Federal de Viçosa - Zona da Mata de Minas Gerais. Revista Árvore, v. 24, n. 4, p. 397-405, 2000. 
SOARES JÚNIOR, F. J. Composição florística e estrutura da vegetação arbórea de um fragmento de Floresta Estacional Semidecidual na Fazenda Tico-Tico, ViçosaMG. 2000. 64 f. Dissertação (Mestrado em Botânica) Universidade Federal de Viçosa, Viçosa, 2000.

SPURR, S. H. Origin of the concept of forest sucession. Ecology, v. 33, n. 3, p. 426-427, 1952.

SWAINE, M. D.; WHITMORE, T. C. On the definition of ecological species groups in tropical rain forests. Vegetatio, v. 75, p. 81-86, 1988.

TABARELLI, M. A regeneração da floresta atlântica montana. 1997. $104 \mathrm{f}$. Tese (Doutorado em Ecologia) Universidade de São Paulo, São Paulo, 1997.
TABARELLI, M.; MANTOVANI, W. Colonização de clareiras naturais na floresta atlântica no sudeste do Brasil. Revista Brasileira de Botânica, v. 20, n. 1, p. 57-66, 1997.

VIANA, V. M.; TABANEZ, A. J. A.; MARTINEZ, J. L. A. Restauração e manejo de fragmentos florestais. In: CONGRESSO NACIONAL SOBRE ESSÊNCIAS NATIVAS, 2., 1992, Campos do Jordão. Anais... Campos do Jordão: Instituto Florestal/Secretaria de Meio Ambiente, 1992. p. $400-406$.

WHITMORE, T. C. Canopy gaps and the two major groups of forest trees. Ecology, v. 70, n. 3, p. 536-538, 1989. 